Case Report

\title{
The Unexpected Diagnosis of Hepatic Tuberculosis in an Immunocompetent Patient
}

\author{
Nikolaos Garmpis $\left(\mathbb{D},{ }^{1}\right.$ Christos Damaskos $\left(\mathbb{D},{ }^{1}\right.$ Anna Garmpi ${ }^{(D)},{ }^{2}$ Aliki Liakea $\left(\mathbb{D},{ }^{3}\right.$ \\ and Dimitrios Mantas iD 1 \\ ${ }^{1}$ Second Department of Propedeutic Surgery, Laiko General Hospital, Medical School, National and Kapodistrian University \\ of Athens, Athens, Greece \\ ${ }^{2}$ First Propedeutic Internal Medicine Department, Laiko General Hospital, Medical School, National and Kapodistrian University \\ of Athens, Athens, Greece \\ ${ }^{3}$ First Department of Pathology, Medical School, National and Kapodistrian University of Athens, Athens, Greece
}

Correspondence should be addressed to Nikolaos Garmpis; nikosg22@hotmail.com

Received 14 January 2020; Revised 5 July 2020; Accepted 29 September 2020; Published 6 October 2020

Academic Editor: Muthukumaran Rangarajan

Copyright ( $) 2020$ Nikolaos Garmpis et al. This is an open access article distributed under the Creative Commons Attribution License, which permits unrestricted use, distribution, and reproduction in any medium, provided the original work is properly cited.

\begin{abstract}
Background/Aim. Tuberculosis (TB) is a chronic infectious disease which affects millions. The most affected system is the respiratory. Thus, hepatic TB (HTB) without involvement of other organs is not common. Its clinical manifestations are not specific, and both imaging and histopathological findings are necessary for the diagnosis. The differential diagnosis includes primary and metastatic liver malignancies. Our aim is to describe the rare entity of HTB via a case presentation. Patient and Methods. We report a case of a 50-year-old female with abdominal pain, weight loss, fever, and anorexia. All imaging methods described a liver lesion. She underwent right lobe hepatectomy, and the histological evaluation demonstrated granuloma with central caseous necrosis. Results. Seven months postoperatively, the patient remains fit and healthy. Conclusion. HTB is a rare entity with no specific symptoms, signs, and no laboratory nor imaging findings. It can be managed effectively if diagnosed in time or lead to death if left untreated.
\end{abstract}

\section{Introduction}

Tuberculosis $(\mathrm{TB})$ is an infection which mainly affects the lungs. Extrapulmonary disease can occur at $15-20 \%$ of the patients, and less than $1 \%$ of these cases is located to the liver $[1,2]$. Hepatic TB (HTB) can appear as a result of miliary tuberculosis or as a primary localized lesion [3]. Immunosuppressed patients are more likely to develop extrapulmonary disease or HTB [4]. There should be high clinical suspicion towards this entity, as the symptoms and the findings of HTB, such as abdominal pain or jaundice, are not specific, and the delay of diagnoses could lead to end-stage liver failure and death [5]. Last but not least, the diagnosis of HTB is based on histopathological and microbiological findings from the liver biopsy [6]. Herein, we repost a case of an immunocom- petent female with primary HTB without any other sites of infection.

\section{Case Presentation}

A 50-year-old female patient referred to our hospital with a history of mild abdominal pain, located in the epigastrium and in the right upper quadrant, lasting for more than a month. In addition, she complained about weight loss ( $4 \mathrm{~kg}$ during last month), loss of appetite, and low-grade fever. At the time of administration, the patient weighted at $65 \mathrm{~kg}$ with a height of $162 \mathrm{~cm}$ (BMI: $24.77 \mathrm{~kg} / \mathrm{m}^{2}$ ). There were no night sweats, cough, vomiting, nor dysuria. She suffered from hypertension, diabetes mellitus type 2, dyslipidemia, and seizures for which she was treated with the appropriate medication. 


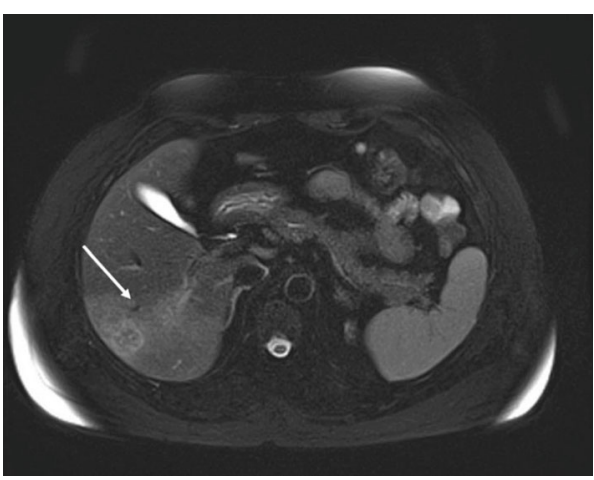

(a)

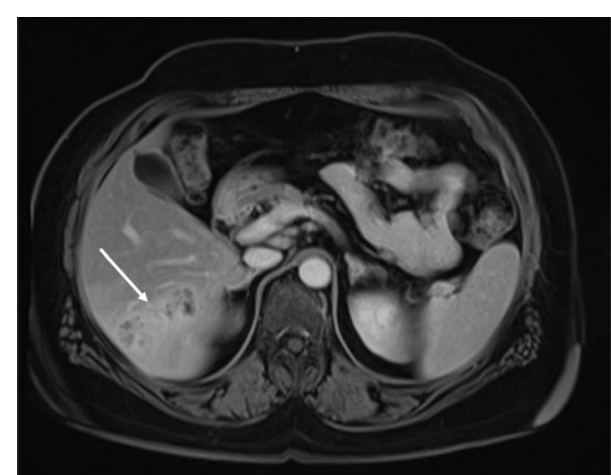

(b)

Figure 1: MRI scan which unveils the hepatic nodular lessions. (a) T1. (b) T2.

During the physical examination, the patient was presented with a blood pressure of $147 / 93 \mathrm{mmHg}$, pulse rate of $78 / \mathrm{min}$, temperature of $37.4^{\circ} \mathrm{C}$, and $17 \mathrm{breaths} / \mathrm{min}$. She appeared pale. There was moderate tenderness of the right upper quadrant with hepatomegaly of three finger-breadths without splenomegaly, jaundice, nor ascites. The physical examination of the thorax did not reveal any abnormality, and no lymphadenopathy was detected.

Complete blood count revealed mild normocyte anemia (hemoglobin $=11.0 \mathrm{~g} / \mathrm{dl}$ ) and an erythrocyte sedimentation rate of $45 \mathrm{~mm} / \mathrm{h}$. Biochemical tests showed elevated alanine transaminase $(355 \mathrm{iU} / \mathrm{L})$, aspartate aminotransferase (339iU/L), and alkaline phosphatase levels (302 iU/L) with normal renal function and electrolytes profile.

The ultrasonography (US) of the abdomen demonstrated a mass in the right lobe of the liver characterized by mixed echogenity. An abdominal computed tomography (CT) followed, which demonstrated a hypodense mass in the right lobe of the liver next to a nonenhanced mass, with normal borders and a size of $1.8 \mathrm{~cm}$, in the right adrenal gland consistent with a benign tumor. Neither chest X-ray nor CT revealed any other abnormal findings. The magnetic resonance imaging (MRI) of the abdomen showed hypointense and hyperintense multiple nodular lesions in T1-weighed imaging and T2 weighed-imaging, respectively, with abnormal borders with a greatest size of $2 \mathrm{~cm}$ (Figure 1). In addition, these lesions demonstrated enhancement after IV contrast administration. The initial differential diagnosis of these radiological findings included primary hepatocellular carcinoma or metastasic liver lesions. As a result, a core biopsy of liver mass with the assistance of ultrasound was conducted. It showed infiltration of predominant eosinoplils, lymphocytes, plasmatocytes, and some giant cells with epitheloid necrotic granulomas (Figure 2). No fungus or acid-fast bacilli were found in the specimen, and a parasite infection was suspected due to eosinophils presence, but it was unable to exclude malignancy or liver pseudotumor. A hepatectomy, including the VI segment of the right lobe of the liver, was performed, and the histopathological examination revealed multiple granulomas with central caseating necrosis. These findings are consistent with tuberculosis even though there were no acid-fast bacilli in Ziehl-Neelsen stain-

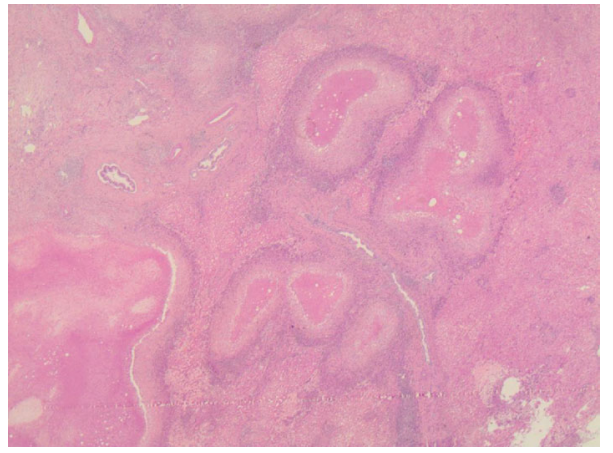

FIGURE 2: Liver section with multiple confluent, caseating granulomas (hematoxylin-eosin, original magnification $\times 20$ ).

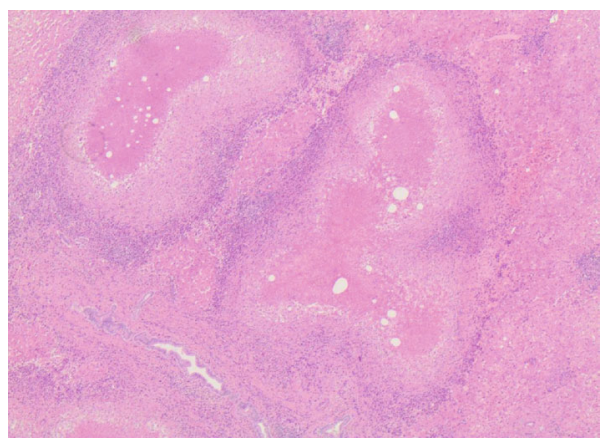

Figure 3: Multiple intrahepatic granulomas that coalesce to form nodules with central necrosis and a surrounding cuff of admixed inflammatory cells (hematoxylin-eosin, original magnification $\times 40$ ).

ing (Figure 3). A QuantiFERON-Tb test was conducted and turned out positive. Lastly, the patient was tested for human immunodeficiency virus (HIV) status, which was negative.

The antituberculous scheme, administrated to the patient, included isoniazid, rifampicin, pyrazinamide, and ethambutol for two months and isoniazid rifampicin for four months more. There were no serious adverse events observed, and all symptoms and laboratory findings were normal after completion of the treatment. Seven months postoperatively, the patient remains fit and healthy. 


\section{Discussion}

Generally, HTB is extremely rare. Its frequency is slightly elevated in Asiatic populations. During the last decade, HTB arises in western countries due to immigration and due to the increasing number of patients with acquired immunodeficiency syndrome (AIDS) [7]. HTB is classified in three types which include the miliary TB derived from generalized infection, the primary hepatic miliary $\mathrm{TB}$, and the rarest nodular lesion named tuberculoma $[8,9]$. It is proposed that the entry point in the liver for the mycobacterium in the tuberculoma is the portal vein and in the miliary HTB is the hepatic artery [10].

The clinical presentation of this entity is not definitive. The most often symptoms and signs are low-grade fever, abdominal pain, tenderness of the right upper quadrant, and hepatomegaly [10]. Our patient did present all the above findings. The laboratory tests usually reveal an elevation of alkaline phosphatase with normal hepatic function, leukocytosis, and anemia [11]. The culture of the mycobacterium and the discovery of acid-fast bacilli are negative in the majority of the times in liver abscesses.

As far as the imaging methods are concerned, the US can demonstrate mostly hypoechoic lesions, while the typical CT finding is the heterogeneity of the lesions which vary from hypodense to hyperdense [12]. On T1-weighted imaging MRI, the lesions are hypointense, while on T2-weighted imaging, the lesions appear isointense and hyperintense with enhancement after contrast administration [13, 14]. In our case, there was a mixed echogenity in the ultrasound, hypodensity on CT, and hypointenisty and hyperintensity on T1- and T2-weighed imaging, respectively.

The diagnosis of HTB requires liver specimen acquired by laparotomy-, US-, or CT-guided biopsy. The diagnosis is confirmed by the presence of a caseating granuloma, or a noncaseating granuloma with positive culture for mycobacterium tuberculosis and/or acid-fast bacilli and improvement with anti-TB therapy [15]. Histologically, eosinophils, plasma cells, lymphohistiocytic cells, and langerhans-type giant cells are encountered in the granuloma [16]. The differential diagnosis includes mainly primary and metastatic liver malignancies.

The appropriate treatment for extrapulmonary $\mathrm{TB}$ is a 6 to 9 months scheme including administration of pyrazinamide, ethambutol, rifampin, and isoniazid for 2 months and afterwards, 4 to 7 months of rifampin and isoniazid [17].

Several articles and case reports in the literature have described interesting cases of isolated hepatic TB or in association with disseminated disease [18-23]. However, only Eshiwe et al. in 2019 [23] have clearly described hepatic involvement to such extent in an immunocompetent patient like in this case. More specifically, Abeysekera et al. [18], Kayar et al. [19], and Sharma et al. [20] described rare cases of isolated hepatic TB in immunocompetent patients. N'goran et al. [21] also described a case of military hepatic TB, and Çalışkan et al. [22] described a case of multiple hypoechoic lesions in a child, but none of them was a severe macronodular type involving the liver.

\section{Conclusion}

In conclusion, HTB is a rare entity with no specific symptoms, signs, laboratory nor imaging findings. Its hallmark is a central caseating necrotic granuloma with or without acid-fast bacilli. Thus, it is of paramount importance to suspect its presence in cases with diagnostic dilemmas and conduct liver biopsy were possible. HTB can be managed effectively if diagnosed in time and it can lead to death if left untreated.

\section{Consent}

The patient's images have been anonymized to maintain privacy.

\section{Conflicts of Interest}

The authors declare that they have no conflict of interest.

\section{Authors' Contributions}

Christos Damaskos and Anna Garmpi performed literature review. Nikolaos Garmpis and Christos Damaskos wrote the manuscript. Dimitrios Mantas provided the clinical input to the case and performed manuscript editing and revision.

\section{References}

[1] A. Mert, R. Ozaras, F. Tabak, R. Ozturk, and M. Bilir, "Localized hepatic tuberculosis," European Journal of Internal Medicine, vol. 14, no. 8, pp. 511-512, 2003.

[2] A. Fanning, "Tuberculosis: 6 extrapulmonary disease," CMAJ, vol. 160, no. 11, pp. 1597-1603, 1999.

[3] R. N. Chien, Y. F. Liaw, and P. Y. Lin, "Hepatic tuberculosis: comparison of miliary and local form," Infection, vol. 23, no. 1, pp. 5-8, 1995.

[4] J. A. Crump, H. O. Ramadhani, A. B. Morrissey et al., "Bacteremic disseminated tuberculosis in sub-saharan Africa: a prospective cohort study," Clinical Infectious Diseases, vol. 55, no. 2, pp. 242-250, 2012.

[5] M. J. Brookes, M. Field, D. M. Dawkins, J. Gearty, and P. Wilson, "Massive primary hepatic tuberculoma mimicking hepatocellular carcinoma in an immunocompetent host," Medscape General Medicine, vol. 8, p. 11, 2006.

[6] T. C. A. Ferrari, C. M. Couto, T. S. Vilaça, and M. A. P. Xavier, "Localized hepatic tuberculosis presenting as fever of unknown origin," The Brazilian Journal of Infectious Diseases, vol. 10, no. 5, pp. 364-367, 2006.

[7] D. N. Amarapurkar, K. B. Chopra, A. Y. Phadke, S. Sahni, S. R. Prabhu, and R. H. Kalro, "Tuberculous abscess of the liver associated with HIV infection," Indian Journal of Gastroenterology, vol. 14, no. 1, pp. 21-22, 1995.

[8] H. D. Rolleston and J. W. McNee, Disease of the Liver, Gall Bladder and Bile Ducts, MacMillan and Co., London, 1929.

[9] C. T. Spiegel and C. U. Tuazon, "Tuberculous liver abscess," Tubercle, vol. 65, no. 2, pp. 127-131, 1984.

[10] S. A. Leader, "Tuberculosis of the liver and gall-bladder with abscess formation: a review and case report," Annals of Internal Medicine, vol. 37, no. 3, pp. 594-606, 1952. 
[11] A. S. Purl, A. K. Nayyar, and J. C. Vij, "Hepatic tuberculosis," Indian Journal of Tubercylosis, vol. 4, pp. 131-134, 1994.

[12] R. S. Yu, S. Z. Zhang, J. J. Wu, and R. F. Li, "Imaging diagnosis of 12 patients with hepatic tuberculosis," World Journal of Gastroenterology, vol. 10, no. 11, pp. 1639-1642, 2004.

[13] Z. M. Fan, Q. Y. Zeng, J. W. Huo et al., "Macronodular multiorgans tuberculoma: CT and MR appearances," Journal of Gastroenterology, vol. 33, no. 2, pp. 285-288, 1998.

[14] Y. Kawamori, O. Matsui, K. Kitagawa, M. Kadoya, T. Takashima, and T. Yamahana, "Macronodular tuberculoma of the liver: CT and MR findings," American Journal of Roentgenology, vol. 158, no. 2, pp. 311-313, 1992.

[15] B. Maharaj, W. P. Leary, and D. J. Pudifin, "A prospective study of hepatic tuberculosis in 41 black patients," The Quarterly Journal of Medicine, vol. 63, no. 242, pp. 517-522, 1987.

[16] D. E. Alcantara-Payawal, M. Matsumura, Y. Shiratori et al., "Direct detection of Mycobacterium tuberculosis using polymerase chain reaction assay among patients with hepatic granuloma," Journal of Hepatology, vol. 27, no. 4, pp. 620-627, 1997.

[17] M. P. Golden and H. R. Vikram, "Extrapulmonary tuberculosis: an overview," American Family Physician, vol. 72, no. 9, pp. 1761-1768, 2005.

[18] W. Y. Mendis Abeysekera, W. D. Dulantha de Silva, A. P. Ginige, P. Pragatheswaran, S. K. Hewage, and A. S. Kumara Banagala, "An isolated tuberculous liver abscess in a nonimmunocompromised patient," Journal of the College of Physicians and Surgeons-Pakistan, vol. 23, no. 9, pp. 667-669, 2013.

[19] Y. Kayar, I. Ekinci, F. T. Turkdogan, M. Atay, R. B. Soytas, and N. B. Kayar, "A rare case of isolated macronodular hepatic tuberculosis (Tuberculous) in an immunocompetent patient," The Journal of the Pakistan Medical Association, vol. 65, no. 11, pp. 1235-1236, 2015.

[20] S. Sharma, K. Mahajan Rakesh, H. K. Gupta, P. Chaskar, and C. Hans, "Tuberculous liver abscess in an immunocompetent adult male-a rare presentation," The Journal of Communicable Diseases, vol. 44, no. 3, pp. 185-188, 2012.

[21] K. N'goran, E. Akaffou, A. N. Konan, and A.-M. N'goanDomoua, "Miliary hepatic: an unusual ultrasound presentation of liver tuberculosis discover in an immuno-competent adolescent," The Pan African Medical Journal, vol. 9, p. 1, 2011.

[22] B. Çalışkan, A. Somer, N. Hatipoğlu et al., "Tuberculous liver abscess in an immunocompetent child with pulmonary tuberculosis as a cause of fever of unknown origin," The Turkish Journal of Pediatrics, vol. 57, no. 1, pp. 85-89, 2015.

[23] C. Eshiwe, F. Shahi, N. Gordon, and P. Lillie, "Rare and unusual case of hepatic and disseminated tuberculosis in an immunocompetent patient," BMJ Case Reports, vol. 12, no. 6, article e229384, 2019. 